COMPARING APPROACHES TO RESEARCH IN GLOBAL AND INTERNATIONAL HEALTH

Appendix A: Characteristics of global health and international health journals included in journal selection process

Global Health

\begin{tabular}{|c|c|c|c|c|c|c|c|}
\hline Journal name & Affiliation & Publisher & $\begin{array}{l}\text { Impact } \\
\text { factor }\end{array}$ & $\begin{array}{l}\text { 5-year } \\
\text { impact } \\
\text { factor }\end{array}$ & Open access & $\begin{array}{l}\text { Frequency of } \\
\text { publication }\end{array}$ & $\begin{array}{l}\text { Financial support for } \\
\text { publication fees }\end{array}$ \\
\hline $\begin{array}{l}\text { Journal of } \\
\text { Global Health } \\
\text { (JoGH) }\end{array}$ & $\begin{array}{l}\text { Edinburgh } \\
\text { University }\end{array}$ & $\begin{array}{l}\text { Edinburgh } \\
\text { University Global } \\
\text { Health Society } \\
\text { (UK) }\end{array}$ & $\begin{array}{l}2.804 \\
(2016) / \\
4.195 \\
(2017)\end{array}$ & N/A & $\begin{array}{l}\text { Yes (\$1200 } \\
\text { fee for } \\
\text { research } \\
\text { articles and } \\
\$ 600 \text { for } \\
\text { non-solicited } \\
\text { viewpoints) }\end{array}$ & Twice a year & $\begin{array}{l}\text { Fees waived for authors } \\
\text { from developing countries } \\
\text { without sufficient funds }\end{array}$ \\
\hline The Lancet & N/A & Elsevier & 17.686 & 18.248 & Yes & Monthly & Authors whose main funder \\
\hline
\end{tabular}


COMPARING APPROACHES TO RESEARCH IN GLOBAL AND INTERNATIONAL HEALTH

\begin{tabular}{|c|c|c|c|c|c|c|c|}
\hline Global Health & & (Netherlands) & (2016) & (2016) & & & $\begin{array}{l}\text { is located either in group A } \\
\text { or B countries of the Health } \\
\text { Inter Network Access to } \\
\text { Research Initiative } \\
\text { (HINARI) or in a country } \\
\text { with a low UNDP human } \\
\text { development index are } \\
\text { exempt }\end{array}$ \\
\hline $\begin{array}{l}\text { Global Health } \\
\text { Promotion }\end{array}$ & $\begin{array}{l}\text { International } \\
\text { Union for } \\
\text { Health } \\
\text { Promotion } \\
\text { and } \\
\text { Education }\end{array}$ & $\begin{array}{l}\text { SAGE Journals } \\
\text { (UK) }\end{array}$ & $\begin{array}{l}1.442 \\
(2016)\end{array}$ & N/A & $\begin{array}{l}\text { OnlineFirst } \\
\text { (forthcoming } \\
\text { articles are } \\
\text { published } \\
\text { online before } \\
\text { they are } \\
\text { scheduled to } \\
\text { appear in } \\
\text { print) }\end{array}$ & Quarterly & Not offered \\
\hline Global Health & $\mathrm{N} / \mathrm{A}$ & BMC part of & N/A & N/A & Yes & No schedule & Authors based in countries \\
\hline
\end{tabular}


COMPARING APPROACHES TO RESEARCH IN GLOBAL AND INTERNATIONAL HEALTH

\begin{tabular}{|c|c|c|c|c|c|c|c|}
\hline $\begin{array}{l}\text { Research and } \\
\text { Policy }\end{array}$ & & Springer Nature & & & & & $\begin{array}{l}\text { classified as LIC or LMIC } \\
\text { by World Bank may } \\
\text { request waiver or discount }\end{array}$ \\
\hline $\begin{array}{l}\text { Annals of } \\
\text { Global Health } \\
\text { (previously } \\
\text { The Mount } \\
\text { Sinai Journal } \\
\text { of Medicine) }\end{array}$ & N/A & $\begin{array}{l}\text { Levy Library Press } \\
\text { (UK) }\end{array}$ & 1.833 & N/A & Hybrid & 6 times a year & $\begin{array}{l}\text { Authors without funds to } \\
\text { pay may request for } \\
\text { discount or full waiver }\end{array}$ \\
\hline $\begin{array}{l}\text { Journal of } \\
\text { Epidemiology } \\
\text { and Global } \\
\text { Health }\end{array}$ & $\begin{array}{l}\text { Saudi } \\
\text { Ministry of } \\
\text { Health }\end{array}$ & $\begin{array}{l}\text { Elsevier } \\
\text { (Netherlands) }\end{array}$ & $\begin{array}{l}0.95 \\
\text { (2015; } \\
\text { calculated } \\
\text { by } \\
\text { Research }\end{array}$ & N/A & Yes & Quarterly & $\begin{array}{l}\text { Corresponding author from } \\
\text { an institution based in } \\
\text { countries in Group A and } \\
\text { Group B of the Hinari list } \\
\text { may receive full waiver of }\end{array}$ \\
\hline
\end{tabular}


COMPARING APPROACHES TO RESEARCH IN GLOBAL AND INTERNATIONAL HEALTH

\begin{tabular}{|c|c|c|c|c|c|c|c|}
\hline & & & Gate) & & & & $50 \%$ discount, respectively \\
\hline $\begin{array}{l}\text { Journal of } \\
\text { Global Health } \\
\text { Perspectives }\end{array}$ & N/A & $\begin{array}{l}\text { First Aid } \\
\text { WorldWide (Menlo } \\
\text { Park, CA) }\end{array}$ & N/A & N/A & Yes & No schedule & Not reported \\
\hline $\begin{array}{l}\text { Global Health: } \\
\text { Science and } \\
\text { Practice } \\
\text { (GHSP) }\end{array}$ & $\begin{array}{l}\text { Supported by } \\
\text { USAID's } \\
\text { Office of } \\
\text { Population } \\
\text { and } \\
\text { Reproductive } \\
\text { Health, } \\
\text { Bureau for } \\
\text { Global Health }\end{array}$ & $\begin{array}{l}\text { John Hopkins } \\
\text { Center for } \\
\text { Communication } \\
\text { Programs (USA) }\end{array}$ & $\begin{array}{l}2.65 \\
\text { (2015; } \\
\text { calculated } \\
\text { by } \\
\text { Research } \\
\text { Gate) }\end{array}$ & $\mathrm{N} / \mathrm{A}$ & Yes & Quarterly & $\begin{array}{l}\text { No publication fees } \\
\text { charged to authors }\end{array}$ \\
\hline $\begin{array}{l}\text { Integrative } \\
\text { Journal of } \\
\text { Global Health }\end{array}$ & N/A & $\begin{array}{l}\text { Insight Medical } \\
\text { Publishing/iMedPub } \\
\text { LTD (UK) }\end{array}$ & N/A & N/A & Yes & 3 times a year & $\begin{array}{l}\text { No discount or waived fees } \\
\text { policy }\end{array}$ \\
\hline
\end{tabular}


COMPARING APPROACHES TO RESEARCH IN GLOBAL AND INTERNATIONAL HEALTH

\section{International Health}

\begin{tabular}{|c|c|c|c|c|c|c|c|}
\hline Journal name & Affiliation & Publisher & $\begin{array}{l}\text { Impact } \\
\text { factor }\end{array}$ & $\begin{array}{l}5 \text {-year } \\
\text { impact } \\
\text { factor }\end{array}$ & $\begin{array}{l}\text { Open } \\
\text { access }\end{array}$ & $\begin{array}{l}\text { Frequency of } \\
\text { publication }\end{array}$ & $\begin{array}{l}\text { Financial support for } \\
\text { publication fees }\end{array}$ \\
\hline $\begin{array}{l}\text { International } \\
\text { Health }\end{array}$ & $\begin{array}{l}\text { Royal Society } \\
\text { of Tropical } \\
\text { Medicine and } \\
\text { Hygiene }\end{array}$ & $\begin{array}{l}\text { Oxford } \\
\text { Academic/Oxford } \\
\text { University Press } \\
\text { (UK) }\end{array}$ & 1.784 & 1.757 & $\begin{array}{l}\text { Options to } \\
\text { publish open } \\
\text { access } \\
\text { (\$3150 } \\
\text { general } \\
\text { articles; } \\
\text { reduced for } \\
\text { developing } \\
\text { countries) }\end{array}$ & 6 times per year & $\begin{array}{l}\text { Waivers apply for } \\
\text { corresponding authors } \\
\text { from LIC and MIC } \\
\text { and those in genuine } \\
\text { hardship }\end{array}$ \\
\hline $\begin{array}{l}\text { International } \\
\text { Journal Health } \\
\text { Services }\end{array}$ & N/A & $\begin{array}{l}\text { SAGE Journals } \\
\text { (UK) }\end{array}$ & 1.135 & 1.215 & $\begin{array}{l}\text { Some } \\
\text { articles have } \\
\text { open access } \\
\text { but there are } \\
\text { also }\end{array}$ & Quarterly & $\begin{array}{l}\text { No fees payable to } \\
\text { publish }\end{array}$ \\
\hline
\end{tabular}


COMPARING APPROACHES TO RESEARCH IN GLOBAL AND INTERNATIONAL HEALTH

\begin{tabular}{|c|c|c|c|c|c|c|c|}
\hline & & & & & $\begin{array}{l}\text { subscription } \\
\text { options }\end{array}$ & & \\
\hline $\begin{array}{l}\text { Tropical } \\
\text { Medicine \& } \\
\text { International } \\
\text { Health }\end{array}$ & $\begin{array}{l}\text { London } \\
\text { School of } \\
\text { Hygiene \& } \\
\text { Tropical } \\
\text { Medicine, } \\
\text { Swiss } \\
\text { Tropical and } \\
\text { Public Health } \\
\text { Institute, } \\
\text { Foundation } \\
\text { Tropical } \\
\text { Health } \\
\text { Medicine and } \\
\text { International } \\
\text { Amsterdamerlands, }\end{array}$ & $\begin{array}{l}\text { John Wiley \& } \\
\text { Sons (founded in } \\
\text { USA but } \\
\text { distribution } \\
\text { worldwide) }\end{array}$ & 2.85 & N/A & $\begin{array}{l}\text { Yes option } \\
\text { available }\end{array}$ & Monthly & $\begin{array}{l}\text { No page charges for } \\
\text { all authors }\end{array}$ \\
\hline
\end{tabular}


COMPARING APPROACHES TO RESEARCH IN GLOBAL AND INTERNATIONAL HEALTH

\begin{tabular}{|c|c|c|c|c|c|c|c|}
\hline & $\begin{array}{l}\text { Belgian } \\
\text { Institute of } \\
\text { Tropical } \\
\text { Medicine and } \\
\text { Bernhard- } \\
\text { Nocht- } \\
\text { Institute for } \\
\text { Tropical } \\
\text { Medicine }\end{array}$ & & & & & & \\
\hline $\begin{array}{l}\text { International } \\
\text { Journal of } \\
\text { Healthcare }\end{array}$ & N/A & $\begin{array}{l}\text { Sciedu Press } \\
\text { (Canada) }\end{array}$ & N/A & N/A & Yes & Twice a year & $\begin{array}{l}\text { No waived fees or } \\
\text { discounts available }\end{array}$ \\
\hline $\begin{array}{l}\text { International } \\
\text { Journal of } \\
\text { Health Policy } \\
\text { and } \\
\text { Management }\end{array}$ & N/A & $\begin{array}{l}\text { Kerman } \\
\text { University of } \\
\text { Medical Sciences } \\
\text { (Iran) }\end{array}$ & $\begin{array}{l}1.36 \text { (2015; } \\
\text { from } \\
\text { Research } \\
\text { Gate) }\end{array}$ & N/A & Yes & Monthly & $\begin{array}{l}\text { No submission or } \\
\text { publication fees }\end{array}$ \\
\hline International & N/A & Taylor \& Francis & 0.39 (2015; & N/A & Yes & Quarterly & No publication fees or \\
\hline
\end{tabular}


COMPARING APPROACHES TO RESEARCH IN GLOBAL AND INTERNATIONAL HEALTH

\begin{tabular}{|c|c|c|c|c|c|c|c|}
\hline $\begin{array}{l}\text { Journal of } \\
\text { Healthcare } \\
\text { Management }\end{array}$ & & $\begin{array}{l}\text { Online (England } \\
\text { \& Wales) }\end{array}$ & $\begin{array}{l}\text { from } \\
\text { Research } \\
\text { Gate) }\end{array}$ & & & & $\begin{array}{l}\text { page charges for } \\
\text { journal }\end{array}$ \\
\hline $\begin{array}{l}\text { International } \\
\text { Journal of } \\
\text { Medical } \\
\text { Sciences }\end{array}$ & N/A & $\begin{array}{l}\text { Ivyspring } \\
\text { International } \\
\text { Publisher } \\
\text { (Australia) }\end{array}$ & 0.517 & N/A & Yes & Monthly & $\begin{array}{l}\text { No policy on discount } \\
\text { or waived publication } \\
\text { fees }\end{array}$ \\
\hline
\end{tabular}

